\title{
Preoperative Versus Postoperative Use of Transversus Abdominis Plane Block With Nonliposomal Bupivacaine on Postoperative Narcotic Use: A Retrospective Study.
}

\author{
Richard Kalu \\ Henry Ford Health System \\ Peter Boateng \\ Henry Ford Health System \\ Lauren Carrier \\ Henry Ford Health System \\ Jaime Garzon \\ Henry Ford Health System \\ Amy Tang \\ Henry Ford Health System \\ Craig Reickert \\ Henry Ford Health System \\ Amalia Stefanou ( $\square$ astefan2@hfhs.org) \\ Henry Ford Health System
}

\section{Research Article}

Keywords: transversus abdominis plane block, preemptive analgesia, TAP block.

Posted Date: January 4th, 2021

DOI: https://doi.org/10.21203/rs.3.rs-131723/v1

License: (c) (1) This work is licensed under a Creative Commons Attribution 4.0 International License. Read Full License

Version of Record: A version of this preprint was published at BMC Anesthesiology on April 12th, 2021. See the published version at https://doi.org/10.1186/s12871-021-01333-6. 


\section{Abstract}

Background: Enhanced recovery protocols optimize pain control via multimodal approaches that include transversus abdominis plane (TAP) block. The aim of this study was to evaluate the effect of preoperative vs postoperative nonliposomal bupivacaine TAP block on postoperative narcotic use after colorectal surgery.

Methods: A retrospective cohort study comparing postoperative narcotic use in patients who received preoperative $(n=240)$ vs postoperative $(n=22)$ nonliposomal TAP blocks. The study was conducted in a single tertiary care institution and included patients who underwent colorectal resections between August 2018 and January 2020. The study measured narcotics use in the postoperative and follow-up periods, operative details, length of stay, reoperation, and readmission rates.

Results: Patients who received postoperative nonliposomal bupivacaine TAP blocks were less likely to require postoperative patient-controlled analgesia (PCA) $(59.1 \%$ vs $83.3 \% ; p=0.012)$ and opioid medications on discharge $(6.4 \%$ vs $16.9 \% ; p=0.004)$ relative to patients who received preoperative TAP. When needed, a significantly smaller amount of opioid was prescribed to the postoperative group (84.5 vs $32.0 \mathrm{mg}, p=0.047)$. No significant differences were noted in the duration of postoperative PCA use, amount of oral narcotic use, and length of stay.

Conclusions: Nonliposomal bupivacaine TAP block administered postoperatively was associated with significantly lower need for postoperative PCA and discharge narcotics. Given the overall short length of stay for the 2 groups, further study is needed to justify the additional cost of liposomal bupivacaine TAP blocks for patients undergoing colorectal resections.

\section{Background}

Enhanced recovery protocols (ERP) after surgery have the aim of reducing morbidity and the surgical stress response while advancing early return of patients to their baseline functioning [1]. Components of ERP include optimal pain control and surgical stress reduction with regional anesthesia, early mobilization, and early enteral nutrition [2, 3]. Multiple studies including randomized controlled trials have shown a reduction in hospital length of stay, duration of postoperative ileus, reduced morbidity, and an earlier return of normal function after ERP implementation [1, 4-6].

Optimal pain management is an integral part of an ERP, particularly after colon surgery. Poor pain control may lead to longer length of stay, cost, and patient dissatisfaction. Most ERPs use a multimodal approach to achieve an optimal pain control, employing neuraxial and regional anesthesia techniques and lower utilization of opioids as the primary analgesic. Transversus abdominis plane (TAP) block is an example of a locoregional anesthetic technique that has been used extensively in abdominal surgery [7, 8]. The TAP block involves injecting bupivacaine into the fascial plane between the internal oblique and transversus abdominis muscles. The duration of action for nonliposomal bupivacaine ranges from 2 to 
10 hours with peak effect noted around 30 to 45 minutes [9]. Although liposomal bupivacaine significantly increases the duration of action, it also adds extra cost.

A TAP block may be administered any time during the immediate perioperative period. However, whether nonliposomal bupivacaine TAP block is effective in colorectal surgery remains to be elucidated. There have been no studies assessing the timing of TAP block administration for optimal postoperative pain control. In the present study, we assessed the effect of timing (preoperative vs postoperative) of TAP block using plain bupivacaine on postoperative narcotic use in patients undergoing colorectal surgery.

\section{Methods}

\section{Patient Selection}

All patients who received TAP blocks during a transabdominal colorectal resection between August 2018 and January 2020 were identified through hospital chart review. The TAP procedure was performed by the regional anesthesiologist on duty that day. All included patients had undergone colorectal resection done by colon and rectal surgeons at our tertiary care hospital. Patient demographics, medical comorbidities, preoperative diagnosis, past medical and surgical history, procedure-related details, and postoperative narcotics use were abstracted. The study was approved by the Henry Ford Health System institutional review board. This manuscript was conducted in accordance with the ethical standards laid down in the amended 1964 Declaration of Helsinki.

Patients were divided in 2 groups based on timing of TAP block procedure: before (preoperative) or immediately after (postoperative) their surgical procedure.

\section{TAP Block Technique}

Under ultrasound guidance, TAP blocks were performed by, or under supervision of, the attending anesthesiologist who specializes in acute pain management and regional anesthesia. The blocks were performed either 1 hour preoperatively or at the conclusion of the surgery procedure within 30 minutes of reaching the post-anesthesia care unit. The TAP block consisted of bilateral injection of $20 \mathrm{~mL}$ of $0.25 \%$ plain bupivacaine in the fascial plane between the internal oblique and transversus abdominis muscles in the midaxillary line. None of the patients in the 2 groups received other blocks, including epidural or spinal anesthesia. Additional opioids, nonsteroidal anti-inflammatory drugs (e.g., ketorolac or ibuprofen), gamma aminobutyric acid analogues (e.g., gabapentin), muscle relaxants (e.g., methocarbamol), and acetaminophen were ordered on an as-needed basis for pain during the postoperative period until discharge.

\section{Outcomes}

The primary outcomes in this study included incidence, type and amount of postoperative narcotic use, and hospital length of stay after the index surgery. The total amount of opioid and non-opioid analgesic 
medications administered after the index procedure was recorded. Secondary outcomes included procedure-related readmission, reoperation, and opioid refills.

\section{Statistical Analysis}

Statistical analyses were conducted to compare the baseline characteristics of the patients who received preoperative TAP blocks with those who received postoperative TAP blocks. Continuous variables were described using the mean and standard deviation (SD), and categorical variables were described with the frequency and percentage. Analysis of variance or Kruskal-Wallis tests were used for continuous variables and chi-square tests or Fisher's exact tests were used for categorical variables as appropriate. $P$ $<0.05$ was considered statistically significant. All analyses were done in SAS 9.4 (SAS Institute, Cary, NC). Results

\section{Descriptive Analysis}

A total of 262 patients were identified through chart review. A total of 240 patients received preoperative TAP blocks and 22 received postoperative TAP blocks. The mean (SD) patient age was 57.8 years (16 years), $45 \%$ were men, and the mean (SD) body mass index was $28.4 \mathrm{~kg} / \mathrm{m}^{2}\left(7.32 \mathrm{~kg} / \mathrm{m}^{2}\right)$. There were no significant differences in the 2 groups with regard to age, sex, body mass index, American Society of Anesthesiology classification, history of cancer or inflammatory bowel disease, and opioid use at the time of the index procedure (Table 1). The 2 groups also did not differ with regard to history of hypertension, diabetes mellitus, hyperlipidemia, congestive heart failure, chronic pulmonary obstructive disease, smoking, and alcohol use. The surgical indications and surgical approaches were similar between the 2 groups (Table 1). 
Table 1

Patient characteristics based on timing of nonliposomal transversus abdominis plane (TAP) block

\begin{tabular}{|c|c|c|c|c|}
\hline Variables & $\begin{array}{l}\text { Overall } \\
(\mathrm{N}=262)\end{array}$ & $\begin{array}{l}\text { Preoperative } \\
\text { TAP } \\
(n=240)\end{array}$ & $\begin{array}{l}\text { Postoperative } \\
\text { TAP } \\
(n=22)\end{array}$ & $\begin{array}{l}P \text { - } \\
\text { value }\end{array}$ \\
\hline Age, years, mean (SD) & $\begin{array}{l}57.76 \\
(16.10)\end{array}$ & $57.83(15.87)$ & $56.95(18.90)$ & 0.807 \\
\hline Male, no. (\%) & $118(45.0)$ & $107(44.6)$ & $11(50.0)$ & 0.791 \\
\hline $\begin{array}{l}\text { Body mass index, } \mathrm{kg} / \mathrm{m}^{2} \text {, mean } \\
\text { (SD) }\end{array}$ & $28.38(7.32)$ & $28.51(7.48)$ & $27.03(5.27)$ & 0.366 \\
\hline Diabetes mellitus, no. (\%) & $67(25.6)$ & $64(26.7)$ & $3(13.6)$ & 0.278 \\
\hline Hyperlipidemia, no. (\%) & $156(59.5)$ & $144(60.0)$ & $12(54.5)$ & 0.786 \\
\hline Cigarette smoking, no. (\%) & $85(32.4)$ & $80(33.3)$ & $5(22.7)$ & 0.436 \\
\hline History of COPD, no. (\%) & $21(8.0)$ & $19(7.9)$ & $2(9.1)$ & 0.692 \\
\hline Alcohol use, no. (\%) & $126(48.1)$ & $113(47.1)$ & $13(59.1)$ & 0.392 \\
\hline Hypertension, no. (\%) & $132(50.4)$ & $121(50.4)$ & $11(50.0)$ & 0.99 \\
\hline History of CHF, no. (\%) & $22(8.4)$ & $21(8.8)$ & $1(4.5)$ & 0.705 \\
\hline Current opioid use, no. (\%) & $43(16.5)$ & $41(17.2)$ & $2(9.1)$ & 0.499 \\
\hline Steroid use, no. (\%) & $37(14.1)$ & $31(12.9)$ & $6(27.3)$ & 0.126 \\
\hline $\begin{array}{l}\text { Previous abdominal surgery, no. } \\
\text { (\%) }\end{array}$ & $170(64.9)$ & $157(65.4)$ & $13(59.1)$ & 0.718 \\
\hline \multicolumn{5}{|l|}{ Blood thinner use, no. (\%) } \\
\hline Aspirin & $52(19.8)$ & $47(19.6)$ & $5(22.7)$ & 0.941 \\
\hline Warfarin & $7(2.7)$ & $6(2.5)$ & $1(4.5)$ & 0.463 \\
\hline Plavix & $11(4.2)$ & $11(4.6)$ & $0(0.0)$ & 0.607 \\
\hline Others & $25(9.5)$ & $22(9.2)$ & $3(13.6)$ & 0.451 \\
\hline ASA class, no. (\%) & & & & 0.241 \\
\hline ASA class 1 & $1(0.4)$ & $1(0.4)$ & $0(0.0)$ & \\
\hline ASA class 2 & $85(32.4)$ & $78(32.5)$ & $7(31.8)$ & \\
\hline ASA class 3 & $169(64.5)$ & $156(65.0)$ & $13(59.1)$ & \\
\hline
\end{tabular}




\begin{tabular}{|lllll|}
\hline Variables & $\begin{array}{l}\text { Overall } \\
\mathbf{N}=\mathbf{2 6 2})\end{array}$ & $\begin{array}{l}\text { Preoperative } \\
\text { TAP } \\
(\mathbf{n}=\mathbf{2 4 0})\end{array}$ & $\begin{array}{l}\text { Postoperative } \\
\text { TAP } \\
\mathbf{( n = 2 2 )}\end{array}$ & $\begin{array}{c}\boldsymbol{P} \\
\text { value }\end{array}$ \\
\hline ASA class 4 & $7(2.7)$ & $5(2.1)$ & $2(9.1)$ & \\
\hline ASA >2 & $176(67.2)$ & $161(67.1)$ & $15(68.2)$ & 0.99 \\
\hline Surgical indication, no. (\%) & & & & 0.882 \\
\hline Malignancy & $129(49.2)$ & $119(49.6)$ & $10(45.5)$ & 0.401 \\
\hline IBD & $52(19.8)$ & $46(19.2)$ & $6(27.3)$ & 0.738 \\
\hline Benign disease & $128(48.9)$ & $116(48.3)$ & $12(54.5)$ & \\
\hline $\begin{array}{l}\text { ASA, American Society of Anesthesiology; COPD, chronic obstructive pulmonary disease; CHF, } \\
\text { congestive heart failure; IBD, inflammatory bowel disease; SD, standard deviation. }\end{array}$ & \\
\hline
\end{tabular}

\section{Analgesic Requirements}

Table 2 shows the postoperative analgesics used by the two groups. The patients who received nonliposomal bupivacaine TAP blocks postoperatively experienced a statistically significant reduction in the overall use of patient-controlled analgesia (PCA) compared with those who received preoperative TAP blocks ( $59.1 \%$ vs $83.3 \% ; p=0.012)$. The postoperative TAP group was also less likely to be prescribed opioid medication at the time of discharge $(6.4 \%$ vs $16.9 \% ; p=0.004)$. For patients who received prescription opioid at the time of discharge, the patients who had postoperative TAP blocks received a significantly smaller amount of opioid ( $84.5 \mathrm{vs} 32.0 \mathrm{mg} ; p=0.047$ ). There were no differences between the groups with regard to duration of PCA or intravenous and oral narcotics use. 
Table 2

Postoperative analgesics use based on when the nonliposomal TAP block was administered

\begin{tabular}{|c|c|c|c|c|}
\hline Variables & $\begin{array}{l}\text { Overall } \\
(\mathrm{N}=262)\end{array}$ & $\begin{array}{l}\text { Preoperative } \\
\text { TAP } \\
(n=240)\end{array}$ & $\begin{array}{l}\text { Postoperative } \\
\text { TAP } \\
(n=22)\end{array}$ & $\begin{array}{l}P \text { - } \\
\text { value }\end{array}$ \\
\hline PCA use, no. (\%) & $213(81.3)$ & $200(83.3)$ & $13(59.1)$ & 0.012 \\
\hline PCA type & & & & 0.003 \\
\hline Morphine & $172(65.6)$ & $165(68.8)$ & $7(31.8)$ & \\
\hline Hydromorphone & $31(11.8)$ & $26(10.8)$ & $5(22.7)$ & \\
\hline Morphine + hydromorphone & $10(3.8)$ & $9(3.8)$ & $1(4.5)$ & \\
\hline \multicolumn{5}{|l|}{ PCA amount, mg, mean (SD) } \\
\hline Morphine & $\begin{array}{l}27.40 \\
(57.37)\end{array}$ & $\begin{array}{l}27.09 \\
(51.87)\end{array}$ & $\begin{array}{l}30.77 \\
(101.32)\end{array}$ & 0.019 \\
\hline Hydromorphone & $2.42(8.07)$ & $2.36(8.18)$ & $3.17(6.81)$ & 0.156 \\
\hline PCA duration, hours, mean (SD) & $\begin{array}{l}33.97 \\
(64.30)\end{array}$ & $\begin{array}{l}34.66 \\
(66.22)\end{array}$ & $26.36(37.48)$ & 0.263 \\
\hline IV opioid use, no. (\%) & $54(20.6)$ & $46(19.2)$ & $8(36.4)$ & 0.102 \\
\hline IV opioid amount, mg, mean (SD) & $\begin{array}{l}1.80 \\
(10.39)\end{array}$ & $1.38(9.07)$ & $6.42(19.50)$ & 0.051 \\
\hline Oral opioid use, no. (\%) & $215(82.1)$ & $197(82.1)$ & $18(81.8)$ & 0.99 \\
\hline $\begin{array}{l}\text { Oral opioid amount, morphine equivalents, } \\
\text { mean (SD) }\end{array}$ & $\begin{array}{l}93.94 \\
(153.61)\end{array}$ & $\begin{array}{l}87.71 \\
(117.82)\end{array}$ & $\begin{array}{l}161.93 \\
(360.53)\end{array}$ & 0.743 \\
\hline Discharge opioid, mg, mean (SD) & $\begin{array}{l}82.35 \\
(82.01)\end{array}$ & $\begin{array}{l}85.40 \\
(84.52)\end{array}$ & 49.09 (32.02) & 0.047 \\
\hline $\begin{array}{l}\text { Discharge opioid, morphine equivalents, } \\
\text { mean (SD) }\end{array}$ & $\begin{array}{l}123.52 \\
(123.01)\end{array}$ & $\begin{array}{l}128.09 \\
(126.78)\end{array}$ & $73.64(48.03)$ & 0.005 \\
\hline $\begin{array}{l}\text { Prescription muscle relaxants on discharge, } \\
\text { no. (\%) }\end{array}$ & $108(41.4)$ & $97(40.6)$ & $11(50.0)$ & 0.528 \\
\hline $\begin{array}{l}\text { Opioid use on first postoperative follow-up, } \\
\text { no. }(\%)\end{array}$ & $19(7.3)$ & $18(7.5)$ & $1(4.5)$ & 0.99 \\
\hline $\begin{array}{l}\text { Opioid refill request at first postoperative } \\
\text { follow up, no. (\%) }\end{array}$ & $3(1.1)$ & $2(0.8)$ & $1(4.5)$ & 0.605 \\
\hline
\end{tabular}

Procedure-Related Details, Length of Stay, Reoperation, and Readmission 
Table 3 presents the procedure-related details, length of stay, and reoperation and readmission rates for the 2 groups. Surgical approach did not differ based on timing of the locoregional anesthesia. There was no statistically significant difference in procedure length, estimated blood loss, length of hospital stays, reoperation, or readmission rates between the 2 groups. 
Table 3

Procedure-related details, length of stay, reoperation, and readmission

\begin{tabular}{|c|c|c|c|c|}
\hline Variables & $\begin{array}{l}\text { Overall } \\
(N=262)\end{array}$ & $\begin{array}{l}\text { Preoperative } \\
\text { TAP } \\
(n=240)\end{array}$ & $\begin{array}{l}\text { Postoperative } \\
\text { TAP } \\
(n=22)\end{array}$ & $P$-value \\
\hline Surgical procedure, no. (\%) & & & & 0.554 \\
\hline Surg_type ${ }^{*}$ & $195(74.4)$ & $178(74.2)$ & $17(77.3)$ & \\
\hline Surg_type $^{\dagger}$ & $38(14.5)$ & $36(15.0)$ & $2(9.1)$ & \\
\hline Surg_type $3^{\ddagger}$ & $11(4.2)$ & $9(3.8)$ & $2(9.1)$ & \\
\hline Surg_type4§ & $18(6.9)$ & $17(7.1)$ & $1(4.5)$ & \\
\hline Surgical approach, no. (\%) & & & & 0.439 \\
\hline Open & $69(26.3)$ & $62(25.8)$ & $7(31.8)$ & \\
\hline Laparoscopic & $79(30.2)$ & 75 (31.2) & $4(18.2)$ & \\
\hline Robotic & $114(43.5)$ & $103(42.9)$ & $11(50.0)$ & \\
\hline \multicolumn{5}{|l|}{ Procedure details } \\
\hline Emergent procedure, no. (\%) & $2(0.8)$ & $1(0.4)$ & $1(4.5)$ & 0.161 \\
\hline $\begin{array}{l}\text { Procedure length, minutes } \\
\text { (SD) }\end{array}$ & $208.38(96.27)$ & $207.79(97.16)$ & $214.82(87.76)$ & 0.655 \\
\hline $\mathrm{EBL}$ in $\mathrm{mL}$, mean (SD) & $\begin{array}{l}100.05 \\
(185.54)\end{array}$ & $100.43(190.96)$ & $95.91(113.14)$ & 0.855 \\
\hline LOS in days, mean (SD) & $4.92(5.95)$ & $4.82(5.62)$ & $6.00(8.87)$ & 0.836 \\
\hline Readmission rate, no. (\%) & $29(11.2)$ & $28(11.8)$ & $1(4.5)$ & 0.485 \\
\hline Reoperation rate, no. (\%) & $5(1.9)$ & $4(1.7)$ & $1(4.5)$ & 0.901 \\
\hline \multicolumn{5}{|c|}{$\begin{array}{l}\text { EBL, estimated blood loss; LOS, length of day; SD, standard deviation; TAP, transversus abdominis } \\
\text { plane. }\end{array}$} \\
\hline \multicolumn{5}{|c|}{$\begin{array}{l}\text { *surg_type1 includes hemicolectomy, sigmoidectomy, low anterior resection, total abdominal } \\
\text { colectomy and abdominoperineal resection }\end{array}$} \\
\hline \multicolumn{5}{|c|}{${ }^{\dagger}$ surg_type2 includes ostomy reversal } \\
\hline \multicolumn{5}{|c|}{${ }^{\ddagger}$ surg_type3 includes ostomy creation } \\
\hline
\end{tabular}




\section{Discussion}

Postoperative pain management is an integral part of achieving the goals of ERPs after colorectal surgery. TAP blocks are an attractive approach for minimizing the use of opioids, especially given their low risk of adverse effects. Although usually done preoperatively, our study showed that postoperative TAP block with nonliposomal bupivacaine appeared to be at least as efficacious as preoperative TAP block in reducing postoperative intravenous narcotics use, both PCA and administered intravenous injections. Furthermore, postoperative nonliposomal TAP block was associated with a reduced total amount of prescription opioids needed to the time of discharge from the hospital. Other variables such as length of stay, estimated operative blood loss, procedure length, reoperation and readmission rates did not differ between preoperative or postoperative administration of the TAP.

The effectiveness and feasibility of TAP blocks in colorectal surgery has been shown in multiple studies [10-15]. In a randomized, placebo-controlled clinical trial, Tikuisis et al. showed that patients who received ropivacaine TAP blocks had significantly lower pain scores at 2, 4, and 12 hours at rest, and at 2 and 4 hours during movement. The TAP group also used significantly less fentanyl and ketorolac following a hand-assisted laparoscopic left hemicolectomy for colon cancer compared to those who received placebo [11]. Pirrera et al. compared the use of preoperative ropivacaine TAP block vs thoracic epidural analgesia in patients before elective laparoscopic colon resection. Both patient groups were a part of a standard enhanced recovery after surgery pathway. Albeit a case-control study, pain control was comparable between the 2 groups. Additionally, the TAP group had significantly lower rates of postoperative nausea, vomiting, ileus, and paresthesia. There was no significant difference in hospital length of stay or 30-day readmission rate. In a prospective, randomized, double-blind study, Keller et al. assessed the effect of TAP blocks on postoperative pain in patients following laparoscopic colorectal resections. Compared to their counterparts, the TAP group had significantly lower pain scores and used fewer opioids. However, there was no difference in hospital length of stay and readmission rate between the groups [15]. These research study findings are consistent with our current results.

Given that TAP block is both feasible and effective for optimizing pain control after colon surgery, it is important to assess whether the additional cost of using liposomal bupivacaine is justified. Liposomal bupivacaine, an extended-release version, increases the duration of action of plain bupivacaine [9]. Past industry sponsored studies have shown liposomal bupivacaine to be more effective than plain bupivacaine in controlling pain after colectomy, resulting in fewer narcotics use, faster return of bowel function, and shorter length of stay [16-18]. However, in a non-sponsored, prospective, randomized double-blind clinical trial, Knudson et al. found no significant difference in hydromorphone PCA use in the first 2 postoperative days for patients who received liposomal vs plain bupivacaine after colorectal resections [19]. Liposomal bupivacaine is more expensive than plain bupivacaine and hence is not readily available on hospital formulary $[8,20]$. For these reasons, it is rational to consider plain bupivacaine and adjustment of timing as another way to maximize pain control. Our study showed that using plain bupivacaine after surgery was not inferior to preoperative administration and may still provide benefit in terms of PCA use and total narcotic used after colectomy. 
Considering the shorter duration of action of plain bupivacaine, the timing of its administration for TAP blocks may provide the needed analgesia while mitigating cost. Preoperative administration is easily performed in the preoperative area and does not affect surgical planning such as ostomy procedures. It is also in line with preemptive analgesia. Oliveira et al., through a meta-analysis that included a variety of abdominopelvic surgeries, reported a greater postoperative pain control, reduced pain at rest, and decreased opioid used with preoperative TAP compared to placebo or no treatment [21]. The majority of the TAP blocks performed in our study were performed preoperatively, but we found benefit with postoperative TAP blocks. While the numbers are small in the postoperative TAP group, the postoperative TAP group did not show inferior pain control compared to preoperative administrated block. In fact, our results showed that plain bupivacaine TAP administered postoperatively led to a reduced postoperative intravenous narcotic use and lesser amount of discharge prescription narcotics.

The study was limited by its retrospective design, the small number of patients who received postoperative nonliposomal bupivacaine TAP block procedures, and by having been performed in a single center. Although the TAP blocks were performed by, or under supervision of, the attending anesthesiologists who specialize in acute pain management and regional anesthesia, we acknowledge the possibility that performer-related differences could lead to differences in pain relief. However, our results showed that some of the additional cost of using liposomal bupivacaine may be offset by performing nonliposomal TAP blocks after the surgical resection. Finally, our study did not directly measure the patient pain scores. Instead, we used the amount of postoperative narcotics used as a surrogate for the adequacy of pain control. Per our ERP, additional analgesia is prescribed on an asneeded basis and coincides with the patient's assessment of pain on a numeric scale.

\section{Conclusions}

TAP blocks provide an effective and feasible means of optimizing pain control in the era of enhanced recovery after surgery. When administered postoperatively, nonliposomal bupivacaine TAP may be as effective as preoperative TAP blocks, offering the same analgesic effect and minimizing intravenous narcotics use while mitigating the cost of using the liposomal formulation.

\section{Abbreviations}

ERP, enhanced recovery protocols

PCA, patient-controlled analgesia

TAP, transversus abdominis plane

\section{Declarations}

Ethics approval and consent to participate: 
This retrospective chart review research study was categorized as exempt and approved by the Henry Ford Hospital System Institutional Review board (Research Administration, Henry Ford Health System, Detroit, Chairperson Dr. Jonathan Ehrman, IRB \#14286). In addition, the requirement for written informed consent was waived by the Henry Ford Health System Institutional Review board.

Consent for publication: Not applicable.

\section{Availability of data and materials:}

The datasets used and/or analyzed during the current study are not publicly available due to patient privacy and institutional policy but are available from the corresponding author on reasonable request.

Competing interests: The authors declare that they have no competing interests.

Funding: None.

\section{Authors' contributions:}

AT analyzed the patient data obtained and assisted in interpreting the statistical results

PB and LC ensured compliance of the transversus abdominis plane (TAP) block procedure and kept record of patient participants.

RK contributed to the literature review; performed chart reviews of the participants; ensured completeness of data; assisted in interpreting the statistical results; and was a major contributor in writing the manuscript.

JG ensured compliance and consistency of the TAP procedure.

CR reviewed the manuscript for completeness before submission.

AS contributed to the literature review, interpreted that patient data and was a major contributor in writing the manuscript.

All authors read and approved the final manuscript.

Acknowledgements. The authors thank Stephanie Stebens, MLIS, Sladen Library, Henry Ford Hospital, for her additional input in reviewing this manuscript and Karla D Passalacqua, PhD, at Henry Ford Hospital for editorial assistance.

\section{References}

1. Jakobsen DH, Sonne E, Andreasen J, Kehlet H. Convalescence after colonic surgery with fast-track vs conventional care. Colorectal Dis. 2006;8:683-7. 
2. Basse L, Hjort Jakobsen D, Billesbolle P, Werner M, Kehlet H. A clinical pathway to accelerate recovery after colonic resection. Ann Surg. 2000;232:51-7.

3. Kehlet $\mathrm{H}$, Mogensen T. Hospital stay of 2 days after open sigmoidectomy with a multimodal rehabilitation programme. Br J Surg. 1999;86:227-30.

4. Kehlet $H$, Dahl JB. Anaesthesia, surgery, and challenges in postoperative recovery. Lancet. 2003;362:1921-8.

5. Khoo CK, Vickery CJ, Forsyth N, Vinall NS, Eyre-Brook IA. A prospective randomized controlled trial of multimodal perioperative management protocol in patients undergoing elective colorectal resection for cancer. Ann Surg. 2007;245:867-72.

6. Muller S, Zalunardo MP, Hubner M, Clavien PA, Demartines N, Zurich Fast Track Study Group. A fasttrack program reduces complications and length of hospital stay after open colonic surgery. Gastroenterology. 2009;136:842-7.

7. Charlton S, Cyna AM, Middleton P, Griffiths JD. Perioperative transversus abdominis plane (TAP) blocks for analgesia after abdominal surgery. Cochrane Database Syst Rev. 2010:Cd007705.

8. Felling DR, Jackson MW, Ferraro J, Battaglia MA, Albright JJ, Wu J, et al. Liposomal bupivacaine transversus abdominis plane block versus epidural analgesia in a colon and rectal surgery enhanced recovery pathway: a randomized clinical trial. Dis Colon Rectum. 2018;61:1196-204.

9. Beiranvand S, Moradkhani MR. Bupivacaine versus liposomal bupivacaine for pain control. Drug Res (Stuttg). 2018;68:365-9.

10. Stokes AL, Adhikary SD, Quintili A, Puleo FJ, Choi CS, Hollenbeak CS, et al. Liposomal bupivacaine use in transversus abdominis plane blocks reduces pain and postoperative intravenous opioid requirement after colorectal surgery. Dis Colon Rectum. 2017;60:170-7.

11. Tikuisis R, Miliauskas P, Lukoseviciene V, Samalavicius N, Dulskas A, Zabuliene L, et al. Transversus abdominis plane block for postoperative pain relief after hand-assisted laparoscopic colon surgery: a randomized, placebo-controlled clinical trial. Tech Coloproctol. 2016;20:835-44.

12. Pirrera B, Alagna V, Lucchi A, Berti P, Gabbianelli C, Martorelli G, Mozzoni L, et al. Transversus abdominis plane (TAP) block versus thoracic epidural analgesia (TEA) in laparoscopic colon surgery in the ERAS program. Surg Endosc. 2018;32:376-82.

13. Conaghan P, Maxwell-Armstrong C, Bedforth N, Gornall C, Baxendale B, Hong LL, et al. Efficacy of transversus abdominis plane blocks in laparoscopic colorectal resections. Surg Endosc. 2010;24:2480-4.

14. Smith SR, Draganic B, Pockney P, Holz P, Holmes R, Mcmanus B, et al. Transversus abdominis plane blockade in laparoscopic colorectal surgery: a double-blind randomized clinical trial. Int $\mathrm{J}$ Colorectal Dis. 2015;30:1237-45.

15. Keller DS, Ermlich BO, Schiltz N, Champagne BJ, Reynolds HL, Stein SL, et al. The effect of transversus abdominis plane blocks on postoperative pain in laparoscopic colorectal surgery: a prospective, randomized, double-blind trial. Dis Colon Rectum. 2014;57:1290-7. 
16. Guerra L, Philip S, Lax EA, Smithson L, Pearlman R, Damadi A. Transversus abdominis plane blocks in laparoscopic colorectal surgery: better pain control and patient outcomes with liposomal bupivacaine than bupivacaine. Am Surg. 2019;85:1013-6.

17. Cohen SM. Extended pain relief trial utilizing infiltration of Exparel(®), a long-acting multivesicular liposome formulation of bupivacaine: a phase IV health economic trial in adult patients undergoing open colectomy. J Pain Res. 2012;5:567-72.

18. Marcet JE, Nfonsam VN, Larach S. An extended paln relief trial utilizing the infiltration of a longacting Multivesicular liPosome foRmulation Of bupiVacaine, EXPAREL (IMPROVE): a phase IV health economic trial in adult patients undergoing ileostomy reversal. J Pain Res. 2013;6:549-55.

19. Knudson RA, Dunlavy PW, Franko J, Raman SR, Kraemer SR. Effectiveness of liposomal bupivacaine in colorectal surgery: a pragmatic nonsponsored prospective randomized double blinded trial in a community hospital. Dis Colon Rectum 2016;59:862-9.

20. Kim AJ, Yong RJ, Urman RD. The role of transversus abdominis plane blocks in enhanced recovery after surgery pathways for open and laparoscopic colorectal surgery. J Laparoendosc Adv Surg Tech A. 2017;27:909-14.

21. De Oliveira GS Jr, Castro-Alves LJ, Nader A, Kendall MC, McCarthy RJ. Transversus abdominis plane block to ameliorate postoperative pain outcomes after laparoscopic surgery: a meta-analysis of randomized controlled trials. Anesth Analg. 2014;118:454-63. 